\title{
Efficacy of Percutaneous Endoscopic Gastrostomy for Patients With Esophageal Cancer During Preoperative Therapy
}

\author{
AKIRA MATSUMOTO, MASAMI YUDA, YUJIRO TANAKA, YUICHIRO TANISHIMA, FUMIAKI YANO, \\ KATSUNORI NISHIKAWA, YOSHIO ISHIBASHI and KATSUHIKO YANAGA \\ Department of Surgery, Jikei University School of Medicine, Tokyo, Japan
}

\begin{abstract}
Background/Aim: This study aimed to clarify the benefits and disadvantages of percutaneous endoscopic gastrostomy (PEG) for patients with esophageal cancer (EC) during preoperative therapy. Materials and Methods: We retrospectively reviewed 92 patients who underwent esophagectomy for EC after preoperative therapy. Patients were divided into the PEG group $(n=14)$ and the control group $(n=78)$ and compared regarding patient characteristics, nutritional status, operative variables, and postoperative complications. Results: In the PEG group first nutritional status and tumor stage were significantly worse, but changes of nutritional status from first visit to operation were significantly better. According to the intraoperative thermal imaging, there was no patient with blood flow disturbance in the gastric conduit due to PEG. Short-term surgical outcomes did not significantly differ. Conclusion: PEG has less adverse effects on gastric tube production in esophagectomy and may be considered in highly selective patients during preoperative therapy.
\end{abstract}

Preoperative chemotherapy or chemoradiotherapy has become standard for patients with locally advanced esophageal cancer (EC), and thus the time from diagnosis to surgery has become elongated $(1,2)$. As patients with locally advanced EC often have dysphagia, and chemotherapy also causes a loss of appetite, they tend to be malnourished at the time of surgery. Therefore, nutritional support during the preoperative period is very important to improve surgical outcome.

Percutaneous endoscopic gastrostomy (PEG) is a useful tool for patients with EC (3). In particular, PEG construction via the introducer technique enables the provision of ideal

Correspondence to: Akira Matsumoto, MD, Ph.D., Department of Surgery, Jikei University School of Medicine, 3-25-8, Nishishinbashi, Minato-ku, Tokyo 105-8461, Japan. Tel: +81 334331111, Fax: +81 354724140, e-mail: ii3a-mtmt@asahi-net.or.jp

Key Words: Esophageal cancer, percutaneous endoscopic gastrostomy, preoperative therapy. nutritional support for patients with $\mathrm{EC}$, and can be performed if the small-diameter endoscope can pass through the stenosis. For such a procedure, there is no need for dilation and there is no danger of PEG site metastasis (4). However, the usefulness of preoperative PEG is unknown, as there is insufficient research into the effect of surgery that damages the stomach to be used for reconstruction and the effect of short-term preoperative nutritional support.

The aim of the present study was to clarify the effect of preoperative PEG on the surgical outcomes and nutritional status of patients with EC.

\section{Materials and Methods}

Nighty-eight consecutive patients with EC underwent esophagectomy after preoperative chemotherapy or chemoradiotherapy at Jikei University School of Medicine, (Tokyo, Japan) between March 2009 and November 2016. Six patients in whom colonic or jejunum conduit was used for reconstruction for reasons not related to PEG placement were excluded, and so a final total of 92 patients were included. All included patients underwent upper gastrointestinal endoscopy, and computed tomography of the neck, chest, and abdomen. All patients were discussed in multidisciplinary team meetings. A nutritional support team managed nutritional replenishment of all patients, and prioritized in order oral intake, tube feeding via PEG, and intravenous nutrition. Only patients with severe dysphagia for solids due to advanced esophageal stricture underwent PEG via the introducer technique $(n=14)$. Patients with locally advanced tumors received chemotherapy or chemoradiotherapy, restaged, and then surgical resection was performed if feasible. Patients underwent surgical resection after 4-8 weeks of preoperative chemotherapy or chemoradiotherapy. For the purpose of the present study, patients were divided into the PEG and control groups. Nutritional status was assessed with measurements of total lymphocyte count (TLC), body mass index (BMI), total protein (TP), serum albumin (Alb), cholinesterase $(\mathrm{ChE})$, and hemoglobin $(\mathrm{Hb})$ levels at the first visit and within 1 week prior to surgery, and the differences between the values at the two timepoints were compared.

At the time of surgery, the gastrostomy tube was removed, and the gastric hole was closed with absorbable sutures under the direct or laparoscopic approach. The fistula of the abdominal wall was excised, and the muscle layer was closed, while the skin holes were left open. For esophageal reconstruction we assessed blood flow in the gastric conduit with thermal imaging in all patients. 
Table I. Clinical background of all 92 patients.

\begin{tabular}{lccc}
\hline & PEG group (n=14) & Control group (n=78) & $p$-Value \\
\hline Gender (M/F) & $12 / 2$ & $68 / 10$ & 0.67 \\
Age, years Mean (range) & $66.7(52-75)$ & $1 / 10 / 33 / 31 / 3$ & 0.37 \\
Tumor site Ce/Ut/Mt/Lt/Ae & $0 / 2 / 7 / 5 / 0$ & & 0.91 \\
Clinical TNM & & $4 / 22 / 48 / 4$ & 0.06 \\
T 1/2/3/4 & $0 / 0 / 12 / 2$ & $12 / 42 / 20 / 3$ & 0.1 \\
N 0/1/2/3 & $0 / 6 / 6 / 2$ & $4 / 28 / 40 / 6$ & 0.03 \\
Clinical stage I/II/III/IV & $0 / 0 / 12 / 2$ & & 0.63 \\
Preoperative therapy & & $8 / 66 / 2 / 2$ & 0.44 \\
FP/DCF/NCRT/d-CRT & $1 / 11 / 1 / 1$ & 71.7 & 0.14 \\
Number of days from treatment start to operation & 74.6 & & 0.89 \\
Esophagectomy technique & \multicolumn{2}{c}{$24 / 54$} & \\
$\quad$ VATS/Open & $1 / 13$ & $7 / 33 / 20 / 12 / 6$ & \\
Pathological response Grade 0/Ia/Ib/II/III & $1 / 7 / 2 / 3 / 1$ & & \\
\hline
\end{tabular}

PEG: Percutaneous endoscopic gastrostomy; FP: chemotherapy with 5-FU and CDDP; DCF: chemotherapy with docetaxel, 5-FU and CDDP; NCRT: neoadjuvant chemo-radiotherapy consisting of 40 Gy radiation with FP; d-CRT: definitive chemo-radiotherapy consisting of radiation above 52 Gy and FP; VATS: video assisted thoracic surgery.

Demographics, tumor location, stage of disease, and nutritional status were assessed. Intraoperative variables consisted of intraoperative blood loss, operative time, surgical approach, and type of conduit. Postoperative variables included total length of hospital stay, 30- and 90-day postoperative mortality rates, and complications that occurred within 30 days postoperatively. Tumor staging was determined in accordance with the International Union Against Cancer TNM classification of malignant tumors ( 7 th edition).

Statistical analysis. Statistical analyses were performed using JMP11 for Windows (SAS Institute, Cary, NC, USA). Comparisons between the two groups were performed by Student's t-test for continuous variables, and $\chi^{2}$ or Fisher's exact test for discrete variables. Statistical significance of the differences expressed as the rate of change between the two timepoints were calculated as analysis of repeated measurement data by multivariate analysis of variance. $p<0.05$ was regarded as statistically significant.

\section{Results}

Fourteen patients underwent PEG constructions, which was performed during preoperative chemotherapy or chemoradiotherapy in four patients, within 1 week before chemotherapy in nine patients, and within 2 weeks before chemotherapy in one patient. There were no complications related to PEG construction. All patients in the PEG group underwent gastric tube reconstruction. In the assessment with intraoperative thermal imaging, there was no patient with blood flow disturbance in the gastric conduit due to PEG. As shown in Figure 1, there was no significant decrease in temperature around PEG hole and its distal side.

The patient characteristics of the two groups are summarized in Table I. The overall mean age was 64 years (range $=52-75$ years). The tumor stage of EC was significantly higher in the PEG group than the control group $(p=0.03)$, and tended to be more advanced in the T category $(p=0.06)$. All patients with stage IV EC had no distant metastases other than in the supraclavicular lymph nodes. Four preoperative treatments were conducted: chemotherapy with 5-FU and CDDP (FP), chemotherapy with docetaxel, 5-FU and CDDP, neoadjuvant chemoradiotherapy with 40 Gy radiation and FP, and definitive chemoradiotherapy consisting of radiation greater than 52 Gy and FP. There were no significant differences between the two groups in the type of preoperative treatment, duration from treatment initiation to surgery, age, sex ratio, tumor site, and esophagectomy technique.

The short-term surgical outcomes are shown in Table II. There were no significant differences between the two groups in any of the assessed outcome measures. In the PEG group, one patient died 66 days postoperatively due to pneumonia.

Nutritional status is shown in Table III. BMI, TP, Alb, $\mathrm{ChE}$, and TLC values at first visit were significantly worse in the PEG group than in the control group. No patient received blood transfusion preoperatively. The change rates of the variables used to indicate the nutritional status are shown in Figure 2. The increase rates of TP, Alb, ChE, and $\mathrm{Hb}$ were significantly better in the PEG group than the control group.

\section{Discussion}

In the current study, we compared the nutritional status during preoperative treatment (chemotherapy or chemoradiotherapy) and the short-term surgical outcomes of patients with EC who underwent PEG versus those who did not. Many of the assessed variables indicated that the PEG group had better nutritional management than the control group, while the 

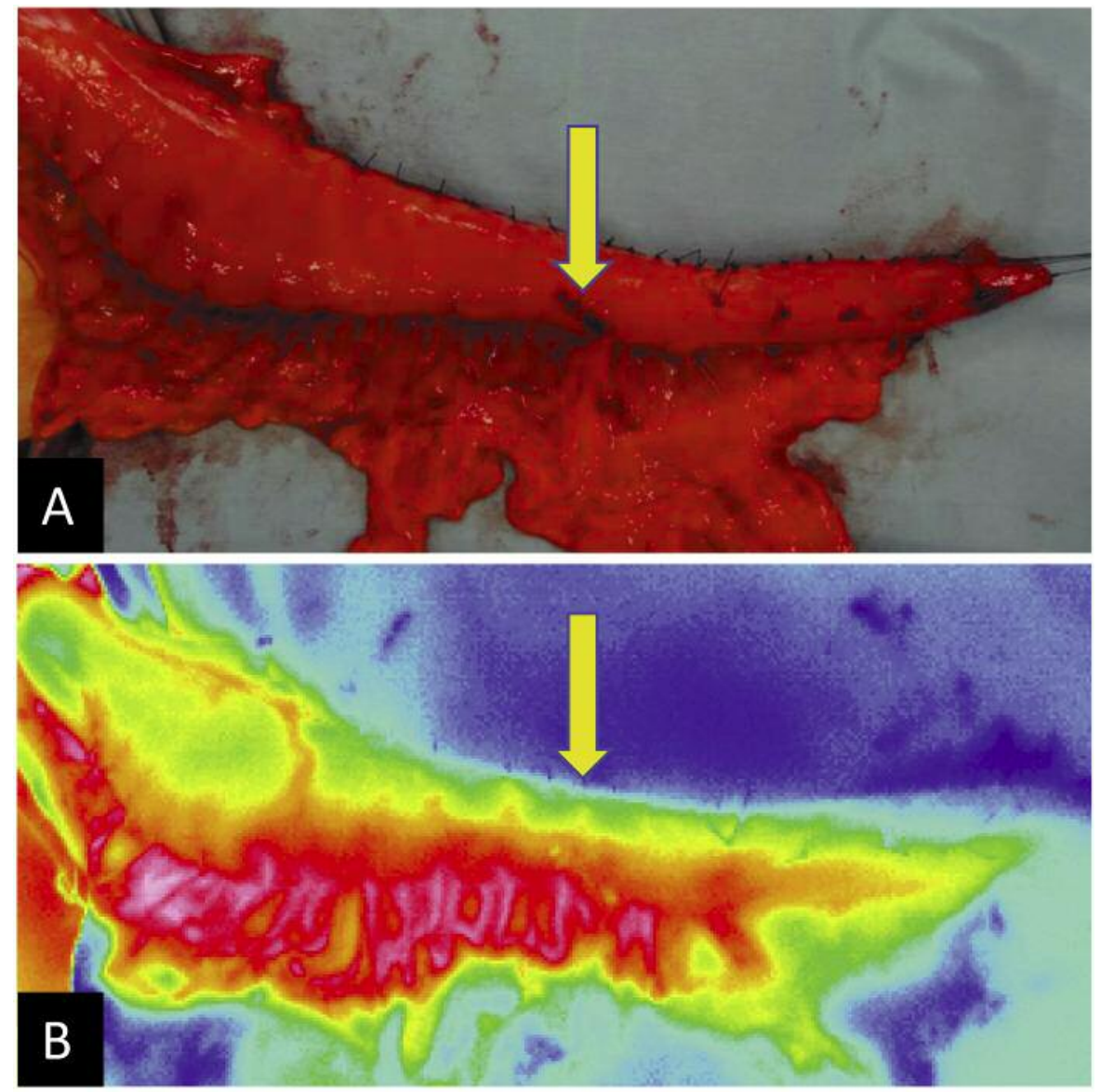

Figure 1. Optical images of the gastric conduit. Naked eye imaging (A), thermal imaging (B). The arrows are where PEG hole closed.

short-term surgical outcomes were similar in both groups.

As patients with advanced EC have a poor prognosis with surgery alone, preoperative chemotherapy or chemoradiotherapy has become standard $(1,2)$. Therefore, the period from the first visit to the time of surgery has become prolonged, and patients with esophageal stricture could become malnourished by the time surgery is performed. For such patients, various methods have been employed to improve the nutritional status. Naso-enteral feeding is the least invasive of these methods, but has issues associated with accidental extubation, misplacement, cosmetic appearance, and discomfort (5). Endoscopic stent placement is associated with a high success rate for the relief of dysphagia, but it also has high rates of stent migration (32\%) and chest discomfort (52\%), and its oncologic safety has not been sufficiently studied (6). Feeding via jejunostomy is a viable option, but this method requires laparotomy, and so it takes some time before preoperative chemotherapy or chemoradiotherapy can be initiated. PEG is a less invasive therapy, and has little influence on preoperative chemotherapy or chemoradiotherapy treatment. In the current study, we did not perform PEG construction while the patients were exhibiting marked adverse effects of chemotherapy, and four patients were able to undergo PEG construction in parallel with preoperative chemotherapy. PEG construction was safely performed without delaying preoperative chemotherapy.

There have been several reports of PEG site metastasis, most of which have occurred after PEG construction via the pull technique (7-9). As the gastrostomy device does not pass through the stenosis, PEG construction via the introducer technique can be performed if a small-diameter endoscope can pass through the stenosis, which can abrogate the risk of metastasis. Similarly, PEG construction via the introducer technique is associated with a relatively reduced risk of peristomal infection (10). If there is cancer or stenosis in the pathway of the endoscope, we routinely perform PEG via the introducer technique, as in the patients in the current study.

Thrombosis of the right gastroepiploic artery, which makes the use of a gastric conduit unsuitable, has been reported in individual cases (11). However, some studies report that PEG has no adverse effects on surgery $(12,13)$. 

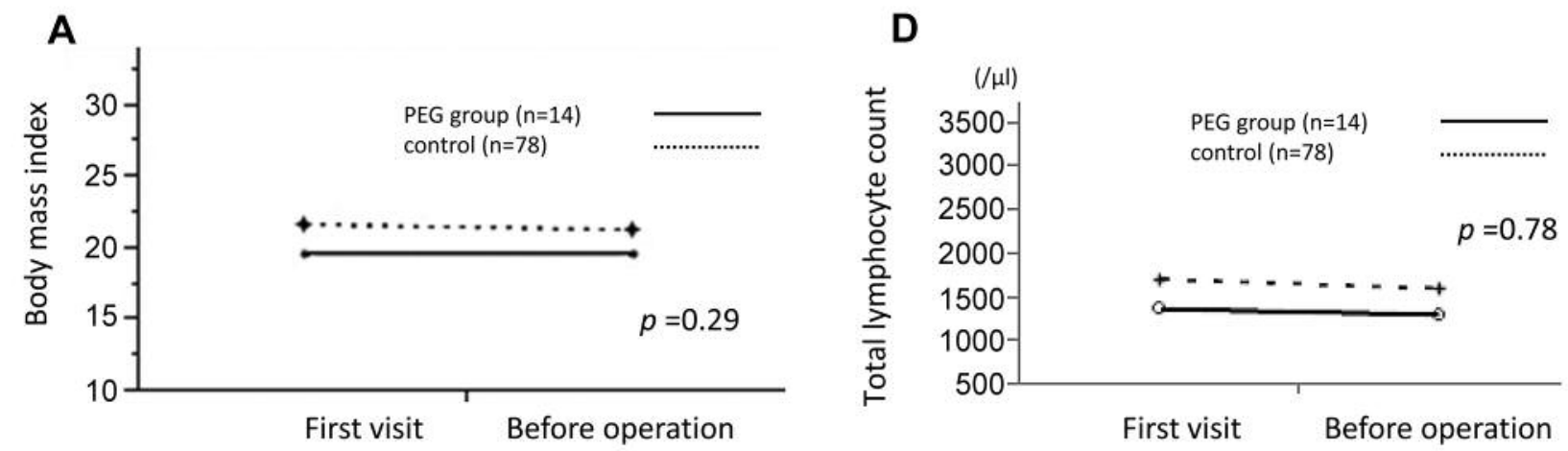

\section{B}
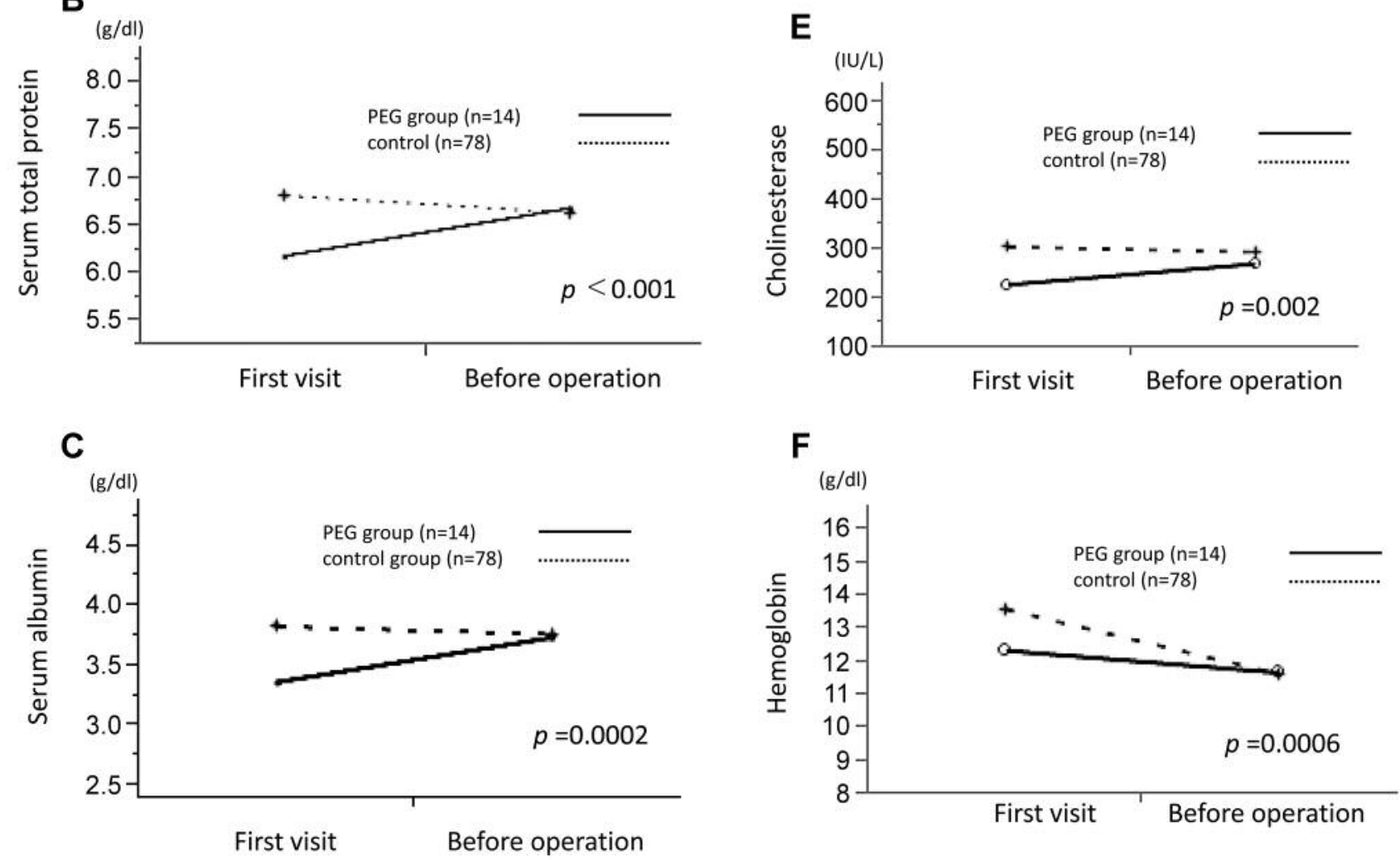

Figure 2. Changes in the assessed nutritional status indicators. Body mass index (A), serum total protein (B), serum albumin (C), total lymphocyte count $(D)$, serum cholinesterase $(E)$, hemoglobin $(F)$.

Similarly, in the current study, there were no significant differences between PEG and control groups in short-term surgical outcomes. Moreover, blood flow was assessed in the gastric conduit with thermal imaging in all patients and there was no patient with blood flow disturbance. We have previously reported that thermal imaging is superior in quantitative assessment of blood flow in the gastric conduit (14). Therefore, we showed that PEG has less adverse effect on gastric tube production in blood flow. The surgical procedure resulting from PEG construction was relatively easy (even via the laparoscope technique), and could be completed in approximately 20 min. Furthermore, wound infection was minimized by resecting the fistula and keeping the skin hole open.

There have been few reports on the effect of PEG on nutritional supplementation during the limited period when preoperative chemotherapy or chemoradiotherapy treatments are being performed. In the current study, the PEG group had significantly more advanced EC and worse nutritional status than the control group, as PEG construction was performed only in patients with advanced esophageal stenosis. Nevertheless, by the time of surgery, many nutritional 
Table II. Short-term results of surgery.

\begin{tabular}{lccc}
\hline & PEG group (n=14) & Control group (n=78) & $p$-Value \\
\hline The median of the operative time (min) & 576 & 628 & 0.67 \\
The median of bleeding (ml) & 580 & 405 & 0.26 \\
The median of postoperative hospitalization (days) & 26.5 & 25 & 0.69 \\
Postoperative complications & & & \\
Surgical site infection & $5(35.7 \%)$ & $14(17.9 \%)$ & 0.94 \\
Leakage & $3(21.4 \%)$ & $20(25.6 \%)$ & 0.85 \\
Stricture & $3(21.4 \%)$ & 0 & 0.73 \\
90 Days Mortality & $1(7.1 \%)$ & 0 & $\mathrm{NS}$ \\
30 Days Mortality & 0 & $\mathrm{NS}$ \\
\hline
\end{tabular}

PEG: Percutaneous endoscopic gastrostomy; NS: not significant.

Table III. Nutritional status of two groups.

\begin{tabular}{|c|c|c|c|}
\hline & PEG group $(n=14)$ & Control group $(\mathrm{n}=78)$ & $p$-Value \\
\hline \multicolumn{4}{|c|}{ Mean of body mass index } \\
\hline At first visit & 19.8 & 21.8 & 0.02 \\
\hline Before operation & 19.7 & 21.4 & 0.06 \\
\hline \multicolumn{4}{|c|}{ Mean of total protein $(\mathrm{g} / \mathrm{dl})$} \\
\hline At first visit & 6.19 & 6.82 & $<0.0001$ \\
\hline Before operation & 6.69 & 6.65 & 0.75 \\
\hline \multicolumn{4}{|c|}{ Mean of albumin $(\mathrm{g} / \mathrm{dl})$} \\
\hline At first visit & 3.37 & 3.83 & 0.0005 \\
\hline Before operation & 3.74 & 3.77 & 0.79 \\
\hline \multicolumn{4}{|c|}{ Mean of cholinesterase (IU/l) } \\
\hline At first visit & 229 & 306 & 0.001 \\
\hline Before operation & 271 & 295 & 0.25 \\
\hline \multicolumn{4}{|c|}{ Mean of total lymphocyte count $(/ \mu l)$} \\
\hline At first visit & 1385 & 1728 & 0.03 \\
\hline Before operation & 1321 & 1620 & 0.08 \\
\hline \multicolumn{4}{|c|}{ Mean of hemoglobin $(\mathrm{g} / \mathrm{dl})$} \\
\hline At first visit & 12.4 & 13.6 & 0.07 \\
\hline Before operation & 11.7 & 11.6 & 0.9 \\
\hline
\end{tabular}

PEG: Percutaneous endoscopic gastrostomy.

indicators in the PEG group had recovered to the same levels as in the control group. This confirms that it is useful to conduct nutrition management by PEG in the short-term during preoperative treatment for patients with esophageal stricture due to EC. Preoperative chemotherapy or chemoradiotherapy treatment alone has reportedly improved the esophageal stricture and nutritional status in some patients (15). However, in patients with advanced stricture due to EC, the stricture often progresses further during preoperative chemotherapy or chemoradiotherapy treatment. Therefore, such patients may benefit from preoperative PEG to achieve effective nutritional support.

There are several limitations in this study. This was a retrospective study, and the choice of the treatment was case-oriented. A prospective cohort study is needed to further evaluate the efficacy of preoperative PEG. Otherwise, a randomized controlled trial comparing preoperative PEG, naso-enteral feeding, endoscopic stent placement, and other nutritional support methods may need to be performed.

In conclusion, PEG is a useful nutritional support method during preoperative chemotherapy or chemoradiotherapy treatment for selected patients with advanced esophageal stricture due to EC and has less adverse effect on gastric tube production.

\section{Conflicts of Interest}

Akira Matsumoto and the other co-authors have no conflicts of interest regarding this study. 


\section{Acknowledgements}

The Authors would like to thank Dr. Kelly Zammit, BVSc, from Edanz Group (www.edanzediting.com/ac), for editing a draft of this manuscript.

\section{Authors' Contributions}

Akira Matsumoto designed the study, and wrote the initial draft of the manuscript. Masami Yuda, Yujiro Tanaka and Yuichiro Tanishima have contributed to data collection and interpretation, and critically reviewed the manuscript. Fumiaki Yano, Katsunori Nishikawa, Yoshio Ishibashi and Katsuhiko Yanaga contributed to analysis and interpretation of data, and assisted in the preparation of the manuscript.

\section{References}

1 Ando N, Kato H, Igaki H, Shinoda M, Ozawa S, Shimizu H, Nakamura T, Yabusaki H, Aoyama N, Kurita A, Ikeda K, Kanda T, Tsujinaka T, Nakamura $\mathrm{K}$ and Fukuda $\mathrm{H}$ : A randomized trial comparing postoperative adjuvant chemotherapy with cisplatin and 5-fluorouracil versus preoperative chemotherapy for localized advanced squamous cell carcinoma of the thoracic esophagus (JCOG9907). Ann Surg Oncol 19: 68-74, 2012. PMID: 21879261. DOI: 10.1245/s10434-011-2049-9

2 van Hagen P, Hulshof MC, van Lanschot JJ, Steyerberg EW, van Berge Henegouwen MI, Wijnhoven BP, Richel DJ, Nieuwenhuijzen GA, Hospers GA, Bonenkamp JJ, Cuesta MA, Blaisse RJ, Busch OR, ten Kate FJ, Creemers GJ, Punt CJ, Plukker JT, Verheul HM, Spillenaar Bilgen EJ, van Dekken H, van der Sangen MJ, Rozema T, Biermann K, Beukema JC, Piet AH, van Rij CM, Reinders JG, Tilanus HW, van der Gaast A and CROSS Group: Preoperative chemoradiotherapy for esophageal or junctional cancer. N Engl J Med 366: 2074-2084, 2012. PMID: 22646630. DOI: 10.1056/NEJMoa1112088

3 Margolis M, Alexander P, Trachiotis GD, Gharagozloo F, and Lipman T: Percutaneous endoscopic gastrostomy before multimodality therapy in patients with esophageal cancer. Ann Thorac Surg 76: 1694-1697; discussion 1697-1698, 2003. PMID: 14602314. DOI: $10.1016 / \mathrm{S} 00034975(02) 04890-7$

4 Yagishita A, Kakushima N, Tanaka M, Takizawa K, Yamaguchi $\mathrm{Y}$, Matsubayashi $\mathrm{H}$ and Ono $\mathrm{H}$ : Percutaneous endoscopic gastrostomy using the direct method for aerodigestive cancer patients. Eur J Gastroenterol Hepatol 24: 77-81, 2012. PMID: 22081009. DOI: 10.1097/MEG.0b013e32834dfd67

5 Pearce CB and Duncan HD: Enteral feeding. Nasogastric, nasojejunal, percutaneous endoscopic gastrostomy, or jejunostomy: its indications and limitations. Postgrad Med J 78: 198-204, 2002. PMID: 11930022. DOI: 10.1136/pmj.78.918.198

6 Nagaraja V, Cox MR and Eslick GD: Safety implications of oesophageal stents used for the palliation of dysphagia in patients undergoing neoadjuvant therapy for oesophageal malignancy-authors' reply. J Gastrointest Oncol 5: E56-57, 2014. PMID: 25083306. DOI: 10.3978/j.issn.2078-6891.2014.043
7 Volkmer K, Meyer T, Sailer M and Fein M: Implantation of an esophageal squamous cell carcinoma at the site of a percutaneous endoscopic gastrostomy. Endoscopy 39: E240-241, 2007. PMID: 17957609. DOI: 10.1055/s-2007-966793

8 Cruz I, Mamel JJ, Brady PG and Cass-Garcia M: Incidence of abdominal wall metastasis complicating PEG tube placement in untreated head and neck cancer. Gastrointest Endosc 62: 708711; quiz 752, 753, 2005. PMID: 16246684. DOI: 10.1016/ j.gie.2005.06.041

9 Cappell MS: Risk factors and risk reduction of malignant seeding of the percutaneous endoscopic gastrostomy track from pharyngoesophageal malignancy: a review of all 44 known reported cases. Am J Gastroenterol 102: 1307-1311, 2007. PMID: 17488255. DOI: 10.1111/j.1572-0241.2007.01227.x

10 Hiki N, Maetani I, Suzuki Y, Washizawa N, Fukuda T, Yamaguchi $\mathrm{T}$ and Tokyo Standard PEGSG: Reduced risk of peristomal infection of direct percutaneous endoscopic gastrostomy in cancer patients: comparison with the pull percutaneous endoscopic gastrostomy procedure. J Am Coll Surg 207: 737-744, 2008. PMID: 18954787. DOI: 10.1016/ j.jamcollsurg.2008.06.335

11 Ohnmacht GA, Allen MS, Cassivi SD, Deschamps C, Nichols FC, 3rd and Pairolero PC: Percutaneous endoscopic gastrostomy risks rendering the gastric conduit unusable for esophagectomy. Dis Esophagus 19: 311-312, 2006. PMID: 16866867. DOI: 10.1111/j.1442-2050.2006.00588.x

12 Bhatti AB, Rizvi FH, Waheed A, Raza SH, Syed AA, Khattak S and Aasim Yusuf $\mathrm{M}$ : Does prior percutaneous endoscopic gastrostomy alter post-operative outcome after esophagectomy. World J Surg 39: 441-445, 2015. PMID: 25270346. DOI: 10.1007/s00268-014-2812-8

13 Wright GP, Foster SM and Chung MH: Esophagectomy in patients with prior percutaneous endoscopic gastrostomy tube placement. Am J Surg 207: 361-365; discussion 364-365, 2014. PMID: 24418181. DOI: 10.1016/j.amjsurg.2013.10.012

14 Nishikawa K, Fujita T, Yuda M, Tanaka Y, Matsumoto A, Tanishima Y, Hanyu N and Yanaga K: Quantitative assessment of blood flow in the gastric conduit with thermal imaging for esophageal reconstruction. Ann Surg, 2018. PMID: 30601260. DOI: $10.1097 /$ SLA.0000000000003169

15 Sunde B, Ericson J, Kumagai K, Lundell L, Tsai JA, Lindblad M, Rouvelas I, Friesland S, Wang N and Nilsson M: Relief of dysphagia during neoadjuvant treatment for cancer of the esophagus or gastroesophageal junction. Dis Esophagus 29: 442447, 2016. PMID: 25809837. DOI: 10.1111/dote.12352 